\title{
Distributed Agent Consensus-Based Optimal Resource Management for Microgrids
}

DOI:

10.1109/TSTE.2017.2740833

\section{Document Version}

Accepted author manuscript

Link to publication record in Manchester Research Explorer

\section{Citation for published version (APA):}

Zhao, T., \& Ding, Z. (2017). Distributed Agent Consensus-Based Optimal Resource Management for Microgrids. IEEE Transactions on Sustainable Energy, 9(1), 443-452. https://doi.org/10.1109/TSTE.2017.2740833

\section{Published in:}

IEEE Transactions on Sustainable Energy

\section{Citing this paper}

Please note that where the full-text provided on Manchester Research Explorer is the Author Accepted Manuscript or Proof version this may differ from the final Published version. If citing, it is advised that you check and use the publisher's definitive version.

\section{General rights}

Copyright and moral rights for the publications made accessible in the Research Explorer are retained by the authors and/or other copyright owners and it is a condition of accessing publications that users recognise and abide by the legal requirements associated with these rights.

\section{Takedown policy}

If you believe that this document breaches copyright please refer to the University of Manchester's Takedown Procedures [http://man.ac.uk/04Y6Bo] or contact uml.scholarlycommunications@manchester.ac.uk providing relevant details, so we can investigate your claim.

\section{OPEN ACCESS}




\title{
Distributed Agent Consensus-Based Optimal Resource Management for Microgrids
}

\author{
Tianqiao Zhao, Zhengtao Ding, Senior Member, IEEE
}

\begin{abstract}
This paper considers the optimal resource management problem for microgrids. Microgrids provide a promising approach to fulfil challenges of the integration of distributed renewable generations and energy storage systems. However, the resource management in a microgrid encounters the new difficulty, i.e. supply-demand imbalance, caused by the intermittence of renewable sources. Therefore, an optimal solution is proposed to the resource management by enhancing the communication and coordination under a multi-agent system framework. An agent is a participant, for instance, the distributed renewable generator/energy storage system of the microgrid. With this multi-agent system, the distributed optimal solution only utilizes the local information, and interacts with the neighbouring agents. Thus, single-node congestion is avoided since the requirement for a central control centre is eliminated, and it is robust against single-link/node failures. The analysis will show that the proposed solution can solve the resource management problem in an initialization-free manner. Additionally, the proposed strategy can maintain the supply-demand balance under a time-varying supply-demand deviation. The simulation studies are carried out for IEEE 14-bus and 162-bus power systems to validate the effectiveness of the proposed distributed solution.
\end{abstract}

Index Terms-Consensus algorithm, resource management, distributed optimization, microgrid, energy storage systems, multi-agent system

\section{INTRODUCTION}

The intelligent microgrid is a promising solution to integrating various distributed renewable generators (RGs), namely wind turbine (WT) and photovoltaic (PV) power generation units, and energy storage systems (ESSs) within a distributed system to support a flexible and efficient electric network [1]. A microgrid should be able to operate in both grid-connected mode and islanded mode [2]. In the grid-connected mode, a microgrid can maintain supply-demand balance by interacting with the main grid [3]. However, in the islanded mode, the microgrid has to balance the supply-demand individually. Due to the intermittence of WTs and PVs, an islanded microgrid will face new operational and control challenges to the resource management.

A solution is to install fast response ESSs for these nonconventional and intermittent renewable sources [4], [5]. Such a hybrid system consists of load demands, distributed RGs and parallel-connected ESSs as depicted in Fig. 1 [6]. However, the integration of RGs and parallel-connected ESSs is limited by some adverse constraints [7]-[9], such as regulation problem [7] and power flow management [8], [9]. Besides, integrating

T. Zhao and Z. Ding are with the School of Electrical and Electronic Engineering, University of Manchester, Sackville Street Building, Manchester M13 9PL, UK (e-mails: tianqiao.zhao@postgrad.manchester.ac.uk; zhengtao.ding@manchester.ac.uk).

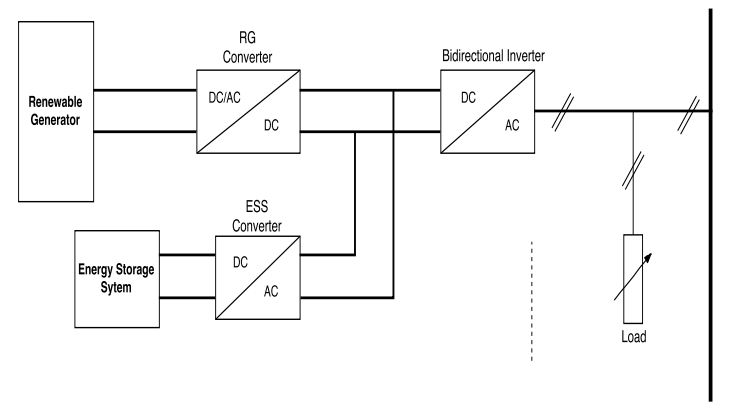

Fig. 1. A diagram of the RG and parallel-connected ESS

ESSs may require additional investment and increase in operation costs with strongly physical restrictions [10]. Since subventions may be restricted in the short-term future, the objective is to reduce generation costs by a proper resource management approach. A resource management problem is to coordinate and optimize the dispatch of RGs and ESSs to meet the microgrid demand. Therefore, in this paper, a distributed solution is designed to optimize the generation cost of all participants, including RGs and the parallel-connected ESSs.

The resource management problem has been widely researched in demand response, economic generation dispatch, and loss minimization with analytical methods [11], [12] or heuristic methods [13], [14]. These centralized methods work effectively for conventional power systems. However, they may lose control efficiency in a microgrid due to the high penetration of RGs. The reason is that a powerful computation centre is indispensable to process numerous collected data from RGs and ESSs in a microgrid [15]. As a result, it brings intractable computation burden, and is sensitive to singlepoint failures. In addition, the participants in the microgrid may be unwilling to release their local information globally such as local cost/utility function and power consumption [16]. The above problems can be avoided by solving the resource management problem in a distributed manner that only utilizes local information through a local private communication network [17]. Besides, an algorithm is proposed to solve the optimal reconfiguration problem in a distributed system [18]. Therefore, it is important to design a distributed solution that can conduce to the microgrid development in a flexible, reliable, and cost-effective way.

Recently, results in the literature have considered distributed solutions to optimal schedule generation supply and load demand [19]-[21]. In [19], the power allocation problem of distributed ESSs was solved by a consensus-based control 
strategy. An external leader is needed to collect and broadcast total supply-demand power mismatch, which makes the control strategy not fully distributed, and is sensitive to the measurement errors of actual power deviations. In [20], a distributed economic operation strategy for a microgrid was proposed to minimize the economical cost through jointly scheduling various participants without considering the single link/node failure. A fully distributed control strategy is proposed in [21]. However, this control strategy relies on a specific initialization procedure during each update step.

Multi-agent system (MAS)-based strategies [22] have been applied to a variety of power system applications, including load restoration [23], frequency regulation [24] and reactive power control [25]. However, the MAS-based solutions in those researches are rule-based designing, and lack of a rigorous stability analysis. The existing distributed solution is proposed under the undirected communication network, which requires a bi-directional communication network, in stead of a possible single-directional communication. In contrast, the scheme with directed information flow would have lower communication cost [26]. Since the of the smart grid is becoming highly scalable due to the integration of smart meters and controllable devices, the scalability of the distributed resource management will be strengthened by introducing the directed communication [27]. Additionally, in a microgrid, although the optimal dispatch problem can run according to the forecasting information, there may exist set-up errors/mismatches during the initialization process. Therefore, a proper designed distributed that can tackle errors in initialization process will be desirable and meaningful for promoting the development of the smart grid.

To address the above issues, we propose an optimal strategy for the resource management in a microgrid, which minimizes the generation cost of all participants in a distributed manner under a directed communication network. With the MAS framework, each agent only needs to exchange information with its neighbours through a directed communication network. As a result, the proposed strategy can work in a distributed manner, and the computational and communication burdens may be reduced by the MAS framework. Besides, by removing a central coordinator, the directed communication is more robust against single-point failures as long as the communication network remains connected. In this paper, by characterizing the the omega-limit set of the trajectories of our strategy, it shows that the power allocation of each RG and parallel-connected ESS can start without the specified initialization and converge to the optimization point while maintaining the supply-demand balance. Furthermore, to overcome the stochastic nature of renewable generations and demands, a simulation study is carried out under a timevarying supply-demand deviation that shows the proposed algorithm converge to the optimal solution while satisfying the equality constraint.

The remainder part of the paper is organized as follows. Section II briefly introduces basic notations and preliminaries. Section III formulates the problem to be solved. Section IV proposes a distributed control strategy to the resource management problem. Section $\mathrm{V}$ presents the simulation results and corresponding analysis. Finally, the conclusion is drawn in Section VI.

\section{NOTATIONS AND PRELIMINARIES}

In this section, we recall some notations and preliminaries that are used in this paper. For $l \in \mathbb{R}$, we denote $\mathcal{H}_{l}=\{x \in$ $\left.\mathbb{R} \mid \mathbf{1}_{n}^{T} x=l\right\}$, and $\mathcal{H}_{0}=\left\{x \in \mathbb{R} \mid \mathbf{1}_{n}^{T} x=0\right\}$, where $\mathbf{1}_{n}=$ $[1,1 \ldots, 1]^{T} \in \mathbb{R}^{n}$. Also, for $B_{0} \in \mathbb{R}^{n}, B_{0}=\left[b_{10}, \ldots, b_{i 0}\right]^{T}$, where $b_{i 0} \neq 0$ denotes $i$ th agent can obtain the information of the total power demand; $b_{i 0}=0$ otherwise; and $\mathbf{1}_{n}^{T} B_{0}=1$.

\section{Graph Theory}

Following [28], [29], we present some basic notions of a directed graph. A directed graph $\mathcal{G}=(\mathcal{V}, \mathcal{E})$, where $\mathcal{V}=\left\{\nu_{1}, \ldots, \nu_{n}\right\}$ denotes the node set and $\mathcal{E} \in \mathcal{V} \times \mathcal{V}$ is the edge set. If $\left(\nu_{i}, \nu_{j}\right) \in \mathcal{E}$ means node $\nu_{i}$ is a neighbour of node $\nu_{j}$. A directed graph contains a directed spanning tree if there exists a root node that has directed paths to all other nodes. A directed graph is strongly connected if there exists a directed path that connects any pair of vertices. For a directed graph $\mathcal{G}$, its adjacency matrix $\mathcal{A}=\left[a_{i j}\right]$ in $\mathbb{R}^{n \times n}$ is defined by $a_{i i}=0, a_{i j}=1$ if $\left(\nu_{j}, \nu_{i}\right) \in \mathcal{E}$ and $a_{i j}=0$ otherwise. A weighted graph $\mathcal{G}=(\mathcal{V}, \mathcal{E}, A)$ consists of a digraph $(\mathcal{V}$, $\mathcal{E}$ ) and an adjacency matrix $\mathcal{A} \in \mathbb{R}_{>0}^{n \times n}$ with $a_{i j}>0$ if and only if $(i, j) \in \mathcal{E}$. The weighted in-degree and out-degree of $i$ are defined as $d_{i n}(i)=\sum_{j=1}^{n} a_{i j}$ and $d_{\text {out }}(i)=\sum_{j=1}^{n} a_{j i}$, respectively. The Laplacian matrix $\mathcal{L}=\left[\mathcal{L}_{i j}\right] \in \mathbb{R}^{n \times n}$ associates with $\mathcal{G}$ is defined as $\mathcal{L}_{i i}=\sum_{j \neq i} a_{i j}$ and $\mathcal{L}_{i j}=-a_{i j}$, $i \neq j . \mathcal{G}$ is defined as weight-balanced if $d_{\text {out }}(\nu)=d_{\text {in }}(\nu)$, for all $\nu \in \mathcal{V}$ iff $\mathbf{1}_{n}^{T} \mathcal{L}=0$ iff $\mathcal{L}+\mathcal{L}^{T}$ is positive semi-definite. If $\mathcal{G}$ is strong connected and weight-balanced, zero is a simple eigenvalue zero of $\mathcal{L}+\mathcal{L}^{T}$. In this case, there is a fact that

$$
x^{T}\left(\mathcal{L}+\mathcal{L}^{T}\right) x \geq \lambda_{2}\left(\mathcal{L}+\mathcal{L}^{T}\right)\left\|x-\frac{1}{n} \mathbf{1}_{n}^{T} \mathbf{1}_{n} x\right\|^{2},
$$

where $\lambda_{2}\left(\mathcal{L}+\mathcal{L}^{T}\right)$ is the smallest non-zero eigenvalue of $\mathcal{L}+$ $\mathcal{L}^{T}$.

\section{PROBLEM Formulation}

The resource management of RGs is formulated to minimize the generation cost while satisfies the supply-demand constraint and the RGs' constraints. In this paper, the objective function considering both RGs and parallel-connected ESSs costs is constructed as

$$
\text { Min } C\left(P_{R}, P_{E}\right)=\sum_{i \in \mathcal{N}_{\mathrm{RG}}} f_{i}\left(P_{R, i}(t)\right)+\sum_{j \in \mathcal{N}_{\mathrm{ESS}}} g_{j}\left(P_{E, j}(t)\right)
$$

where $\mathcal{N}_{\mathrm{RG}}$ and $\mathcal{N}_{\mathrm{ESS}}$ are the sets of RGs and ESSs, respectively. $f_{i}\left(P_{R, i}(t)\right)$ and $g_{j}\left(P_{E, j}(t)\right)$ are the cost function for $i$ th RG and $j$ th ESS, for $i \in \mathcal{N}_{\mathrm{RG}}$ and $j \in \mathcal{N}_{\mathrm{ESS}}$. $P_{R, i}(t)$ and $P_{E, j}(t)$ are the output power of $i$ th $\mathrm{RG}$ and $j$ th ESS. Moreover, define $C_{k}\left(P_{R, i}, P_{E, j}\right)=f_{i}\left(P_{R, i}(t)\right)+$ $g_{j}\left(P_{E, j}(t)\right)$ and $C\left(P_{R}, P_{E}\right)=\sum_{k \in \mathcal{N}} C_{k}\left(P_{R, i}, P_{E, j}\right)$ with $P_{R}=\left[P_{R, i}(t), \ldots, P_{R, n}(t)\right]^{T} \in \mathbb{R}^{n}$ and $P_{E}=$ $\left[P_{E, j}(t), \ldots, P_{R, n}(t)\right]^{T} \in \mathbb{R}^{n}$. 
In this paper, the RG's objective of economic dispatch is to minimize the curtailment of renewable energy in a microgrid. To this end, the cost function of $i$ th RG is expressed as

$$
f_{i}\left(P_{R, i}(t)\right)=a_{i} P_{R, i}(t)^{2}+b_{i} P_{R, i}(t)+c_{i}
$$

where $P_{R, i}^{\max }$ is the predicted maximum power generation capacity of $i$ th RG, and $a_{i}=\frac{\varepsilon_{i}}{2 P_{\max }}, b_{i}=-\varepsilon_{i}$, and $c_{i}=\frac{\varepsilon_{i} P_{\max }}{2}$, respectively. A trade-off factor $\varepsilon_{i}$ is introduced to indicate the between the capacity and generation cost, which can be selected according to the capacity required, the installation and other costs known as 'balance of system cost' for each type of renewable sources [20], [30]. The formulated objective function (3) has a similar format to the cost function in [31], [32] with different the parameter setting. Besides, (3) indicates that the lower power generation cost is realized when the deviation of actual output power $P_{R, i}(t)$ between $P_{R, i}^{\max }$ is minimized. Hence, the minimization of renewable energy curtailment can be derived by minimizing generation cost of RGs [33]. Additional, the following condition is achieved when the incremental rate of each RG is equally, i.e.,

$$
\frac{P_{R, i}(t)}{P_{R, i}^{\max }}=\cdots=\frac{P_{R, k}(t)}{P_{R, k}^{\max }}
$$

which indicates the equal power sharing among RGs.

Following [34], a convex quadratic cost function is adopted to represent the power loss of ESSs during the charging/discharging process, i.e.,

$$
g_{j}\left(P_{E, j}(t)\right)=a_{j} P_{E, j}(t)^{2}+b_{j} P_{E, j}(t)+c_{j}
$$

where $a_{j}, b_{j}$ and $c_{j}$ are the non-negative parameters of $j$ th ESS, which can be selected in terms of the Amoroso and Cappuccino's experimental results [35].

\section{A. Constraints}

The physical limitations on the operation of the studied system are presented as follows:

1) Global Constraint: Since the change of frequency within a microgrid is mainly affected by the supply-demand mismatch, the supply-demand balance should be maintained to ensure the stability of the microgrid. To this end, the active power balance in a microgird can be expressed as

$$
P_{D}(t)=\sum_{i \in \mathcal{N}_{\mathrm{RG}}} P_{R, i}(t)+\sum_{j \in \mathcal{N}_{\mathrm{ESS}}} P_{E, j}(t)
$$

where $P_{D}(t)$ is the total load demand.

2) Local Constraints: The actual output power of each RG and ESS should belong to a feasible range, i.e.,

$$
\begin{aligned}
& P_{R, i}^{\min } \leq P_{R, i} \leq P_{R, i}^{\max } \\
& P_{E, j}^{\min } \leq P_{E, j} \leq P_{E, j}^{\max }
\end{aligned}
$$

where $P_{R, i}^{\min }$ and $P_{R, i}^{\max }$ are the lower and upper bound for $i$ th RG; $P_{E, j}^{\min }$ and $P_{E, j}^{\max }$ are the lower and upper bound for $j$ th ESS.

\section{B. Objective Function}

Considering both the global constraints (6) and local constraints (7), the resource management problem is formulated as

$$
\operatorname{Min} C\left(P_{R}, P_{E}\right)
$$

subject to

$$
\begin{gathered}
P_{D}(t)=\sum_{i \in \mathcal{N}_{\mathrm{RG}}} P_{R, i}(t)+\sum_{j \in \mathcal{N}_{\mathrm{ESS}}} P_{E, j}(t), \\
P_{R, i}^{\min }<P_{R, i}<P_{R, i}^{\max }, \quad \text { for } i \in \mathcal{N}_{R G} \\
P_{E, j}^{\min }<P_{E, j}<P_{E, j}^{\max }, \quad \text { for } j \in \mathcal{N}_{E S S} .
\end{gathered}
$$

Remark 3.1: In the formulation of the objective function, the cost function (8) is used to reflect the operation cost of the islanded microgrid. The formulated cost function consists of a generation cost of each RG and a charging/discharging cost of each BESS. The generation cost of RGs is formulated to minimize the curtailment of renewable energy when the minimized generation cost is achieved. In the meantime, the cost function of BESSs represents the power losses during the charging/discharging process as indicated in [34], which maximizes the actual power output of BESSs while minimizing the power losses. For this reason, the total operation cost will be minimized by the proposed cost function.

The feasibility set and the solution set of the resource management problem is denoted by $\mathcal{F}_{R M}$ and $\mathcal{F}_{R M}^{*}$, respectively. Note that $\mathcal{F}_{R M}^{*}$ is compact since $\mathcal{F}_{R M}$ is compact. Denote $P_{E}=\left[P_{E, 1}, \ldots, P_{E, n}\right] \in \mathbb{R}^{n}$ and $P_{R}=\left[P_{R, 1}, \ldots, P_{R, n}\right] \in$ $\mathbb{R}^{n}$, respectively. A useful Lemma in [36] is introduced as follows

Lemma 3.1: Since $C_{k}\left(P_{R, i}, C_{E, j}\right)$ is convex, locally Lipschitz, and continuously differentiable, the optimization problem has a solution $\left(P_{R}^{*}, P_{E}^{*}\right) \in \mathbb{R}^{n}$ if and only if $\exists \sigma \in \mathbb{R} \quad$ such that $\sigma \mathbf{1}_{n} \in \partial_{P_{R}} C\left(P_{R}, P_{E}\right)$, and $\sigma \mathbf{1}_{n} \in$ $\partial_{P_{E}} C\left(P_{R}, P_{E}\right)$, and $\mathbf{1}_{n} P_{R}^{*}+\mathbf{1}_{n} P_{E}^{*}=P_{D}$.

\section{Distributed SOLUTION OF DYNAMIC ECONOMIC DISPATCH}

The formulated problem (8) is a convex optimization problem with both equality and inequality constraints. Traditional centralized strategies may have some challenges, such as computation burden and non-timely response. In this section, we develop a distributed cooperative strategy for the resource management problem to overcome these challenges, which only utilizes locally available information and interacts with its adjacent agents.

\section{A. Distributed Algorithm Design}

Inspired by the dynamic average consensus estimation method proposed in [37], a distributed solution is developed for the resource management, which allows the power alloca- 
tion of RGs and ESSs to start from any initial condition. The distributed algorithm is formulated as

$$
\begin{aligned}
\dot{P}_{R, i}= & -\sum_{h \in \mathbb{N}_{\mathrm{RG}}} a_{i h}\left(\partial_{P_{R, i}} C_{i}\left(P_{R, i}, P_{E, j}\right)\right. \\
& \left.-\partial_{P_{R, h}} C_{h}\left(P_{R, h}, P_{E, j}\right)\right) \\
\dot{P}_{E, j}= & -\sum_{k \in \mathbb{N}_{\mathrm{ESS}}} a_{j k}\left(\partial_{P_{E, j}} C_{i}\left(P_{R, i}, P_{E, j}\right)\right. \\
& \left.-\partial_{P_{E, k}} C_{k}\left(P_{R, i}, P_{E, k}\right)\right)+\gamma x_{j}, \\
\dot{x}_{j}= & -\beta\left(x_{j}-\left(b_{i 0} P_{D}-P_{E, j}-P_{R, i}\right)\right) \\
& -\alpha \sum_{k \in \mathbb{N}_{\mathrm{ESS}}} a_{j k}\left(x_{j}-x_{k}\right)-\sum_{k \in \mathbb{N}_{\mathrm{ESS}}} a_{j k}\left(\eta_{j}-\eta_{k}\right), \\
\dot{\eta}_{j}= & \alpha \beta x_{j},
\end{aligned}
$$

where $\alpha, \beta, \gamma \in \mathbb{R}_{>0}$ are the parameters to be designed. $\mathbb{N}_{\mathrm{RG}}$ and $\mathbb{N}_{\mathrm{ESS}}$ denotes the neighbour set of $i$ th RG and $j$ th ESS, respectively. In addition, $x_{j}$ is designed to track the difference between supply and demand, $P_{D}-\mathbf{1}_{n}^{T} P_{E}-\mathbf{1}_{n}^{T} P_{R}$. Both RGs and ESSs are deployed through a Laplacian-gradient algorithm to explore the minimization of the generation cost. Meanwhile, we introduce a feedback element, $\gamma x_{j}$ for $j$ th ESS to meet the supply-demand equality condition.

Remark 4.1: Note that the renewable generation may not be considered as dispatchable if they are controlled in maximum peak power tracking (MPPT) mode because of their intermittent and irregular nature. As a result, RGs are not used to compensate the supply-demand mismatch. Thus, only ESSs are deployed to maintain the supply-demand balance in the control design. The feedback term is only introduced for $j$ th ESS to satisfy the supply-demand equality condition. Meanwhile, $i$ th RG only implements (9b) to explore the minimization of its own generation cost.

\section{B. Convergence Analysis}

The algorithm is rewritten in a compact form for convergence analysis as

$$
\begin{aligned}
& \dot{P}_{E}=-\mathcal{L}_{E} \partial_{P_{E}} C\left(P_{R}, P_{E}\right)+\gamma x \\
& \dot{P}_{R}=-\mathcal{L}_{R} \partial_{P_{R}} C\left(P_{R}, P_{E}\right) \\
& \dot{x}=-\alpha \mathcal{L}_{E} x-\beta\left(x-\left(B_{0} P_{D}-P_{E}-P_{R}\right)\right)-\mathcal{L}_{E} \eta \\
& \dot{\eta}=\alpha \beta x
\end{aligned}
$$

where $x, \eta$ are the column vectors containing $x_{i}$, and $\eta_{i}$, respectively. $\mathcal{L}_{E}$ and $\mathcal{L}_{R}$ denote the corresponding Laplacian matrix of network connections of ESSs and RGs, respectively.

Let $P_{k}=P_{R, i}+P_{E, j}$, and $P=\left[P_{1}, \ldots, P_{n}\right] \in \mathbb{R}^{n}$. The $\omega$-limit set of the trajectories of (9) under any initial condition in $\mathbb{R}^{n} \times \mathbb{R}^{n} \times \mathbb{R}^{n}$ is first characterized. Then, with $\mathbb{T}=\mathbb{R}^{n} \times$ $\mathbb{R}^{n} \times \mathbb{R}^{n}$ and $\mathbb{T}_{0}=\mathcal{H}_{P_{D}} \times \mathcal{H}_{0} \times \mathcal{H}_{0}$, the following lemma is introduced.

Lemma 4.1: The $\omega$-limit set of any trajectory of (10) with any initial condition in $\left(P_{k, 0}, x_{0}, \eta_{0}\right) \in \mathbb{T}$ is contained in $\mathbb{T}_{0}$.

Proof: Defining $\phi(t)=\mathbf{1}_{n}^{T} P-P_{D}$, one has

$$
\dot{\phi}(t)=\mathbf{1}_{n}^{T} \gamma x(t)
$$

and

$$
\begin{aligned}
\ddot{\phi}(t) & =\mathbf{1}_{n}^{T} \gamma \dot{x}(t) \\
& =-\mathbf{1}_{n}^{T} \beta \gamma x(t)+\mathbf{1}_{n}^{T} \beta \gamma\left(x-\left(B_{0} P_{D}-P\right)\right) \\
& =-\beta \gamma \phi(t)-\beta \dot{\phi}(t) .
\end{aligned}
$$

The system can be rewritten as a linear system $\dot{z}=A z$ with $z=\left[z_{1}, z_{2}\right]^{T}$, and $z_{1}=\phi(t), z_{2}=\dot{\phi}(t)$. The system matrix is

$$
A=\left[\begin{array}{cc}
0 & 1 \\
-\beta \gamma & -\beta
\end{array}\right] \text {. }
$$

Let $R \in \mathbb{R}^{2 \times 2}$ be

$$
R=\frac{1}{2 \gamma \beta^{2}}\left[\begin{array}{cc}
\beta^{2}+\beta \gamma+(\beta \gamma)^{2} & \beta \\
\beta & 1+\beta \gamma
\end{array}\right],
$$

which satisfies $A^{T} R+R A+I=0$. Define $V_{z}=z^{T} R z$ as a Lyapunov function candidate, and the derivative of $V_{z}$ is

$$
\dot{V}_{z}=-z^{T} z \text {. }
$$

Therefore, it can be deduced that $(\phi(t) ; \dot{\phi}) \rightarrow 0$. Furthermore, $\phi=0$ implies that $\mathbf{1}_{n}^{T} P_{E}(t)+\mathbf{1}_{n}^{T} P_{R}(t) \rightarrow P_{D}$ and $\mathbf{1}_{n}^{T} x(t) \rightarrow 0$. Note that $\mathbf{1}_{n}^{T} \dot{\eta}=0$, as $\mathbf{1}_{n}^{T} x(t) \rightarrow 0$.

Remark 4.2: The proof of Lemma 4.1 establishes the exponential stability of the demand mismatch dynamics. With the Theorem 5.4 in [38], it can ensure the input-to-state stability(ISS). Thus it is robust to arbitrary bounded perturbations. Additionally, from the Lyapunov equation $V_{z}=z^{T} R z$, it follows Theorem 4.6 in [38] that gives the convergence rate as

$$
\|x(t)\| \leq \sqrt{\frac{\lambda_{\max }(R)}{\lambda_{\min }(R)}} e^{\frac{-1}{\lambda_{\max }(R)}}\|x(0)\| .
$$

The demand mismatch dynamics depends on the topology of the communication network. However, as indicated in (15), the convergence rate does not directly depend on the knowledge of the Laplacian matrix.

We are now ready to complete the convergence analysis. By applying (10), the trajectories of actual output power of RGs and their parallel-connected ESSs converge to the solution of the optimization problem.

Theorem 4.1: The trajectories of (10) converge to the solution of the optimal charging problem if $\alpha, \beta, \gamma \in \mathbb{R}_{>0}$ satisfy the following conditions, i.e.,

$$
\frac{\beta \lambda_{\max }\left(\mathcal{L}_{R}^{T} \mathcal{L}_{R}\right)}{2 \lambda_{2}\left(\mathcal{L}_{R}+\mathcal{L}_{R}^{T}\right)} \geq 0
$$

and

$$
\frac{\gamma}{\alpha \beta \lambda_{2}\left(\mathcal{L}_{E}+\mathcal{L}_{E}^{T}\right)}+\frac{\beta \lambda_{\max }\left(\mathcal{L}_{E}^{T} \mathcal{L}_{E}\right)}{2}<\lambda_{2}\left(\mathcal{L}_{E}+\mathcal{L}_{E}^{T}\right) .
$$

Proof: A change of coordinates is performed to shift the equilibrium point of (10) to the origin, i.e., $\hat{\eta}=\mathcal{L}_{E}-$ $\beta\left(B_{0} P_{D}-P_{E}-P_{R}\right)$. Then, the dynamics (10) can be transformed as

$$
\begin{aligned}
& \dot{P}_{E}=-\mathcal{L}_{E} \xi_{1}+\gamma x, \\
& \dot{P}_{R}=-\mathcal{L}_{R} \xi_{2}, \\
& \dot{x}=-\alpha \mathcal{L}_{E} x-\beta\left(x-\left(B_{0} P_{D}-P_{E}-P_{R}\right)\right)-\mathcal{L}_{E} \eta, \\
& \dot{\hat{\eta}}=\alpha \beta \mathcal{L}_{E} x+\gamma \beta x-\beta \mathcal{L}_{E} \xi_{1}-\beta \mathcal{L}_{R} \xi_{2},
\end{aligned}
$$


with $\xi_{1} \in \partial_{P_{E}} C\left(P_{R}, P_{E}\right)$ and $\xi_{2} \in \partial_{P_{R}} C\left(P_{R}, P_{E}\right)$.

Next step is to prove that the trajectories of (10) converge to the optimal solution of the formulated problem in the new coordinates.

Consider a candidate Lyapunov function

$$
V=C\left(P_{R}, P_{E}\right)+\frac{1}{2} \gamma \beta\left\|\theta_{1}\right\|^{2}+\frac{1}{2}\left\|\theta_{2}\right\|^{2} .
$$

where an additional transformation is introduced to more easily identify the candidate Lyapunov function, with $\theta_{1}=x$ and $\theta_{2}=\beta x+\hat{\eta}$.

Then the time derivative of (19) along the trajectories of (10) is given as

$$
\begin{aligned}
\dot{V} & =-\xi_{1}^{T} \mathcal{L}_{E} \xi_{1}+\gamma \xi_{1}^{T} \theta_{1}-\xi_{2}^{T} \mathcal{L}_{R} \xi_{2}-\gamma \alpha \beta \theta_{1}^{T} \mathcal{L}_{E} \theta_{1} \\
& -\beta \theta_{2}^{T} \theta_{2}-\beta \theta_{2}^{T} \mathcal{L}_{E} \xi_{1}-\beta \theta_{2}^{T} \mathcal{L}_{R} \xi_{2} \\
& =-\frac{1}{2} \xi_{1}^{T}\left(\mathcal{L}_{E}+\mathcal{L}_{E}^{T}\right) \xi_{1}+\gamma \xi_{1}^{T} \theta_{1}-\frac{1}{2} \xi_{2}^{T}\left(\mathcal{L}_{R}+\mathcal{L}_{R}^{T}\right) \xi_{2} \\
& -\frac{1}{2} \gamma \alpha \beta \theta_{1}^{T}\left(\mathcal{L}_{E}+\mathcal{L}_{E}^{T}\right) \theta_{1}-\beta\left\|\theta_{2}\right\|^{2}-\beta \theta_{2}^{T} \mathcal{L}_{E} \xi_{1} \\
& -\beta \theta_{2}^{T} \mathcal{L}_{R} \xi_{2}
\end{aligned}
$$

Since the directed graph $\mathcal{G}$ is strongly connected and weightbalanced with the fact that $\mathbf{1}_{n}^{T} \theta_{1}=0$ for $\left(P_{E}, P_{R}, \theta_{1}, \theta_{2}\right) \in$ $\mathbb{T}_{0}$, one has

$$
\begin{aligned}
\dot{V} & \leq-\frac{1}{2} \lambda_{2}\left(\mathcal{L}_{E}+\mathcal{L}_{E}^{T}\right)\left\|\xi_{1}-\frac{1}{n} \mathbf{1}_{n}^{T} \mathbf{1}_{n} \xi_{1}\right\|^{2} \\
& +\gamma\left(\xi_{1}-\frac{1}{n} \mathbf{1}_{n}^{T} \mathbf{1}_{n} \xi_{1}\right)^{T} \theta_{1} \\
& -\frac{1}{2} \lambda_{2}\left(\mathcal{L}_{R}+\mathcal{L}_{R}^{T}\right)\left\|\xi_{2}-\frac{1}{n} \mathbf{1}_{n}^{T} \mathbf{1}_{n} \xi_{2}\right\|^{2}-\beta\left\|\theta_{2}\right\|^{2} \\
& -\frac{1}{2} \gamma \alpha \beta \lambda_{2}\left(\mathcal{L}_{E}+\mathcal{L}_{E}^{T}\right)\left\|\theta_{1}\right\|^{2}-\beta \theta_{2}^{T} \mathcal{L}_{E}\left(\xi_{1}-\frac{1}{n} \mathbf{1}_{n}^{T} \mathbf{1}_{n} \xi_{1}\right) \\
& -\beta \theta_{2}^{T} \mathcal{L}_{R}\left(\xi_{2}-\frac{1}{n} \mathbf{1}_{n}^{T} \mathbf{1}_{n} \xi_{2}\right)
\end{aligned}
$$

Defining $\psi_{1}=\xi_{1}-\frac{1}{n}\left(\mathbf{1}_{n}^{T} \mathbf{1}_{n} \xi_{1}\right), \psi_{2}=\xi_{2}-\frac{1}{n}\left(\mathbf{1}_{n}^{T} \mathbf{1}_{n} \xi_{2}\right)$ and $v_{1}^{T}=\left[\psi_{1}^{T}, \theta_{1}^{T}, \theta_{2}^{T}\right], v_{2}^{T}=\left[\psi_{2}^{T}, \theta_{2}^{T}\right]$, we have

$$
\dot{V} \leq v_{1}^{T} J_{1} v_{1}-v_{2}^{T} J_{2} v_{2},
$$

where

$$
J_{1}=\left[\begin{array}{cc}
-\frac{1}{2} \lambda_{2}\left(\mathcal{L}_{E}+\mathcal{L}_{E}^{T}\right) I_{n} & M_{12}^{T} \\
M_{12} & M_{22}
\end{array}\right],
$$

with $M_{12}^{T}=\left[\begin{array}{ll}\frac{1}{2} \gamma I_{n} & -\frac{1}{2} \beta \mathcal{L}_{E}^{T}\end{array}\right]$. and furthermore, $M_{22}=$ $\left[\begin{array}{cc}-\frac{1}{2} \alpha \beta \gamma \lambda_{2}\left(\mathcal{L}_{E}+\mathcal{L}_{E}^{T}\right) I_{n} & 0 \\ 0 & -\beta I_{n}\end{array}\right]$ and

$$
J_{2}=\left[\begin{array}{cc}
-\frac{1}{2} \lambda_{2}\left(\mathcal{L}_{R}+\mathcal{L}_{R}^{T}\right) I_{n} & -\frac{1}{2} \beta \mathcal{L}_{R}^{T} \\
-\frac{1}{2} \beta \mathcal{L}_{R} & 0
\end{array}\right] .
$$
if

Resorting to the Schur complement, $J_{1}$ is negative definite

$$
-\frac{1}{2} \lambda_{2}\left(\mathcal{L}_{E}+\mathcal{L}_{E}^{T}\right) I_{n}+\frac{\gamma}{2 \alpha \beta \lambda_{2}\left(\mathcal{L}_{E}+\mathcal{L}_{E}^{T}\right)} I_{n}+\frac{\beta}{4} \mathcal{L}_{E}^{T} \mathcal{L}_{E}
$$

is negative definite, and $J_{2}$ is positive definite if

$$
\frac{\beta \lambda_{\max }\left(\mathcal{L}_{R}^{T} \mathcal{L}_{R}\right)}{2 \lambda_{2}\left(\mathcal{L}_{R}+\mathcal{L}_{R}^{T}\right)}
$$

is positive definite, respectively. Furthermore, we can conclude that $\dot{V} \leq 0$ by applying (17) and (16). Hence, with the application of LaSalle Invariance principle, we deduce that $\dot{V}=0$ iff $\psi_{1}=\psi_{2}=\theta_{1}=\theta_{2}=0$, which implies that $\partial_{P_{E}} C\left(P_{R}, P_{E}\right) \in \operatorname{span}\left\{\mathbf{1}_{n}\right\}$ and $\partial_{P_{R}} C\left(P_{R}, P_{E}\right) \in \operatorname{span}\left\{\mathbf{1}_{n}\right\}$. Recalling the Lemma 4.1 and the characterization of optimizers in Lemma 3.1, it indicates $\left(P_{E}^{*}, P_{R}^{*}\right)$ is a solution of the optimization problem.

Remark 4.3: The local inequality constraints are taken into account by applying additional projection operations to each RG and ESSs. As shown in the literatures [39], [40], the projection operation does not affect the convergence analysis of the distributed optimal strategy.

Remark 4.4: The operation limits of the power network, such as voltage and line flow limits, could be able to included in the proposed algorithm with applying the projection method, which may lead to different optimization results. Additionally, the proposed method can also deal with the model of the transmission loss in [41], which assumes each agent could estimate the power loss of the line adjacent to it. With the available estimation value, each agent could add this value to the load quantity, which would lead the network to discover a new optimization result that considers the power loss.

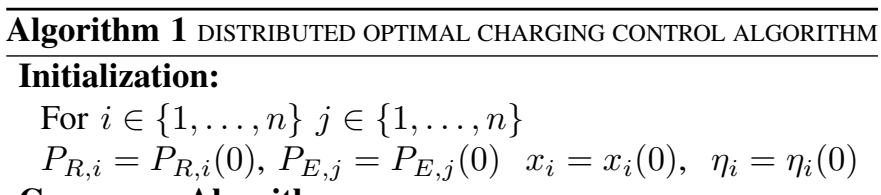

\section{Consensus Algorithm:}

Select $\alpha, \beta, \gamma \in \mathbb{R}_{>0}$

Check If variables satisfy the inequality (16) and (17) in Theorem 4.1,

$$
\left\{\begin{array}{l}
\text { Yes, Flag }=1 \rightarrow \text { Continue } \\
\text { No, Flag }=0 \rightarrow \text { Go back to Select }
\end{array}\right.
$$

\section{Coordination}

RG $i$ and ESS $j$ communicate with its adjacent agents, and update $\left(P_{E, j},, P_{R, i}, x_{j}, \eta_{j}\right)$ according to (10a)-(10d) in Section IV.

End if Each participant achieves the optimal operation.

\section{Algorithm Implementation}

The proposed algorithm can be implemented in a MAS manner, which regards each participant as an agent in the microgrid. A step-by-step algorithm for each agent is shown in Algorithm 1. Each agent equips with two level controls, i.e. top level control and bottom level control as shown in Fig. 2. As indicated in Fig.2, each agent employs both top level control and bottom level control. Since the proposed algorithm is implemented based on the MAS framework, each agent is able to exchange information with its neighbours to complete the determined objective. The proposed algorithm is implemented in the top level to generate the output reference for bottom level control based on the information from both the local measurement and its neighbour's measurement. The proposed algorithm can guarantee the output power reference converge 


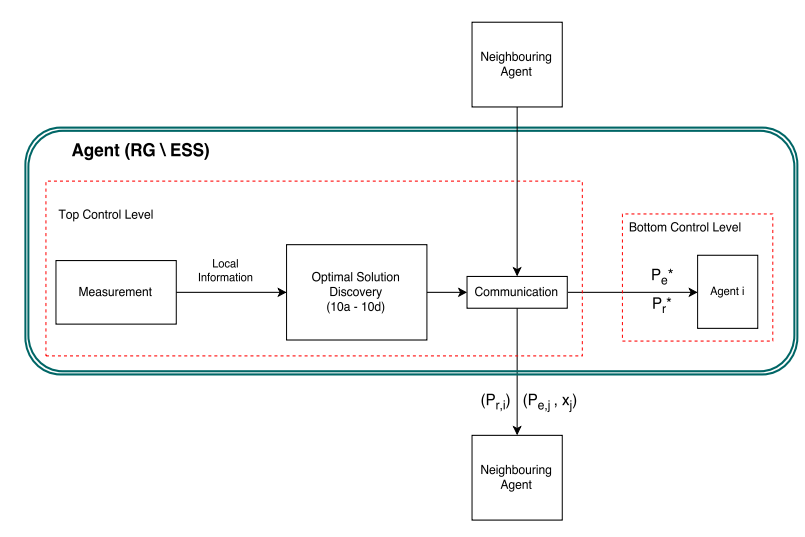

Fig. 2. General operation diagram of an agent.

to the optimal solution as long as there exist an optimal point and communication network is connected. After the reference is generated, the bottom level control will implement the output power reference tracking of each agent. Since the communication network could be independent of the power network [42], a cost-effective communication network could be formed based on the location, convenience of a practical microgrid. While the output power reference is generated, bottom level control is then implemented to control each agent to track this reference.

\section{Simulation Results And Analysis}

In the following case studies, the designed parameters are chosen as $\alpha=14, \beta=0.6, \gamma=3$, which satisfies the condition specified in Theorem 4.1. The parameters of RGs and ESSs are summarized in Table I. The first two cases test the proposed control strategy based on IEEE 14-bus system, and the communication topology is shown as Fig. 3. In Case 5.1, the optimal resource management problem is studied under both constant supply-demand deviation and time-varying deviation conditions. In Case 5.2, a modified five-bus model is applied to compare the effectiveness of the proposed algorithm. Case 5.3 investigates the performance of the proposed strategy under the link/node failures. To accomplish the tractability of the demonstration, it is assumed that if the single-link/node failure occurs in the communication topology, the rest of the weighted-balanced digraph should still remain connected, and thus the remaining nodes would be able to operate with their neighbouring nodes continuously [43]. Finally, the scalability analysis is validated in Case 5.4 in the IEEE 162-bus system with various RGs and ESSs, such as WTs, PVs, Solar thermal, BESSs and fuel cells. Without loss of generality, it is hypothesized that ESS1 knows the total supply-demand mismatch.

\section{A. Case 5.1}

In this case, the performance of the proposed distributed strategy for resource management is investigated in the IEEE 14-bus system. The designed communication network of agents can be independent from the physical bus connections, which is a directed network in this simulation study.

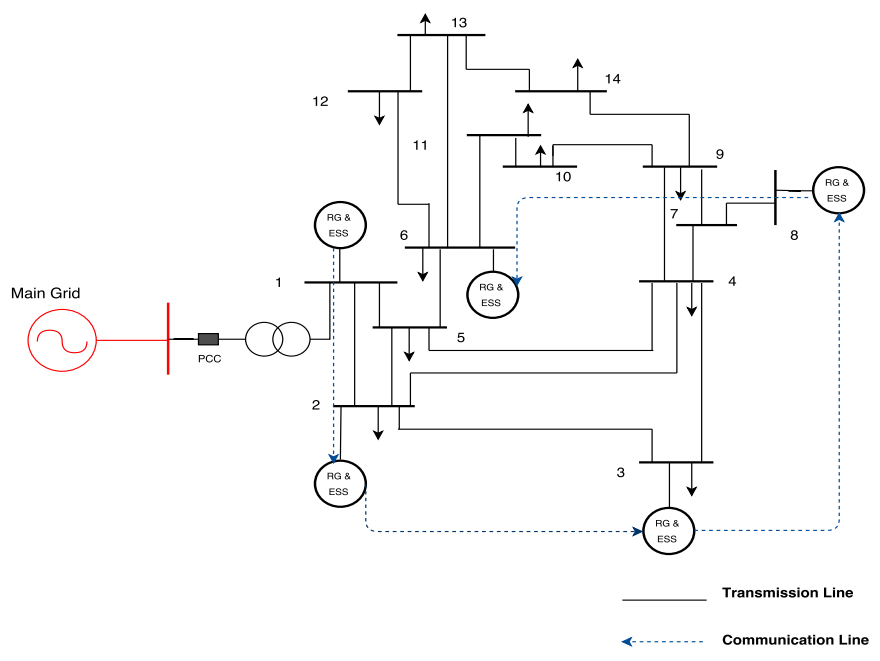

Fig. 3. IEEE 14-bus system.

TABLE I

Simulation PARAMETERS

\begin{tabular}{|c|c|c|c|c|c|c|}
\hline & & $a_{j}$ & $b_{j}$ & $P_{E, j}^{\min }$ (p.u.) & $P_{E, j}^{\max }$ (p.u.) & \\
\hline \multirow{6}{*}{ ESSs } & ESS1 & 0.83 & 0.007 & 0 & 50 & \\
\hline & ESS2 & 0.79 & 0.006 & 0 & 50 & \\
\hline & ESS3 & 0.90 & 0.009 & 0 & 50 & \\
\hline & ESS4 & 0.93 & 0.012 & 0 & 50 & \\
\hline & ESS5 & 0.78 & 0.008 & 0 & 50 & \\
\hline & & \multicolumn{2}{|c|}{$\begin{array}{l}\text { Predicated } \\
\text { Maximum } \\
\text { Capacity }\end{array}$} & $P_{R, i}^{\min }$ (p.u.) & $P_{R, i}^{\max }$ (p.u.) & $\varepsilon_{i}$ \\
\hline \multirow{3}{*}{ PVs } & PV1 & \multicolumn{2}{|c|}{60} & 0 & 60 & 0.1 \\
\hline & PV2 & \multicolumn{2}{|c|}{62} & 0 & 65 & 0.2 \\
\hline & PV3 & \multicolumn{2}{|c|}{65} & 0 & 65 & 0.1 \\
\hline \multirow{2}{*}{ WTs } & WT1 & \multirow{2}{*}{\multicolumn{2}{|c|}{$\begin{array}{l}67 \\
68\end{array}$}} & 0 & 67 & 0.1 \\
\hline & WT2 & & & 0 & 68 & 0.2 \\
\hline
\end{tabular}

1) Constant Supply-demand Mismatch Condition: The total supply-demand mismatch is assumed as 500 p.u.. As shown in Figs. 4 and 5, the power allocations of RGs and parallel-connected ESSs quickly converge to their optimal values, while the supply-demand balance converges to zero, i.e., the optimization objective is achieved.

2) Time-varying Supply-demand Mismatch Condition: if renewable energy resources are controlled by MPPT algorithms, it may cause a time-varying supply-demand imbalance when the available renewable generation cannot meet load demands. As a result, in this subcase, the time-varying imbalance is modelled by a time-varying function, i.e., $P_{D}=500+10 \sin (0.05 t)$ p.u.. The simulation results are shown in Figs. 6 and 7. The allocated actual powers converge to their optimal values, and the deviation between supply and demand power closes to zero.

In this case study, it shows the proposed strategy effectively offsets the supply-demand mismatch, which will help with frequency regulation in an islanded microgrid. 


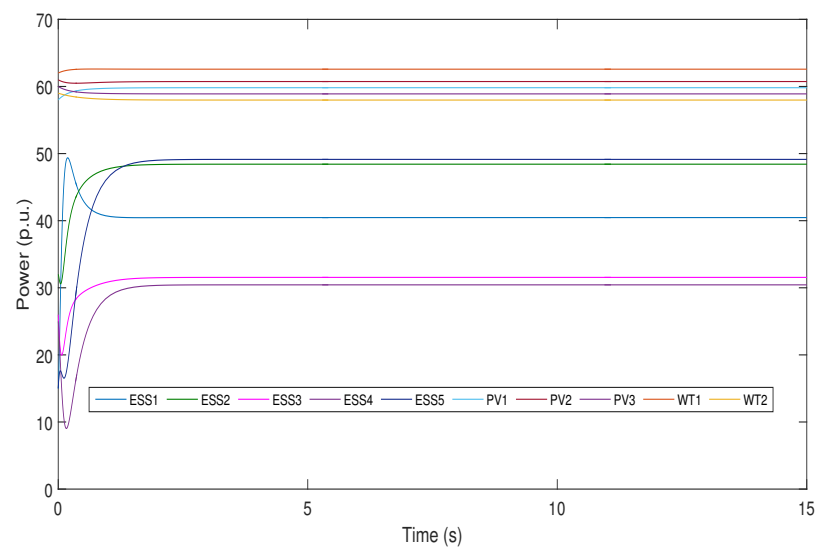

Fig. 4. The actual output power of RGs and ESSs

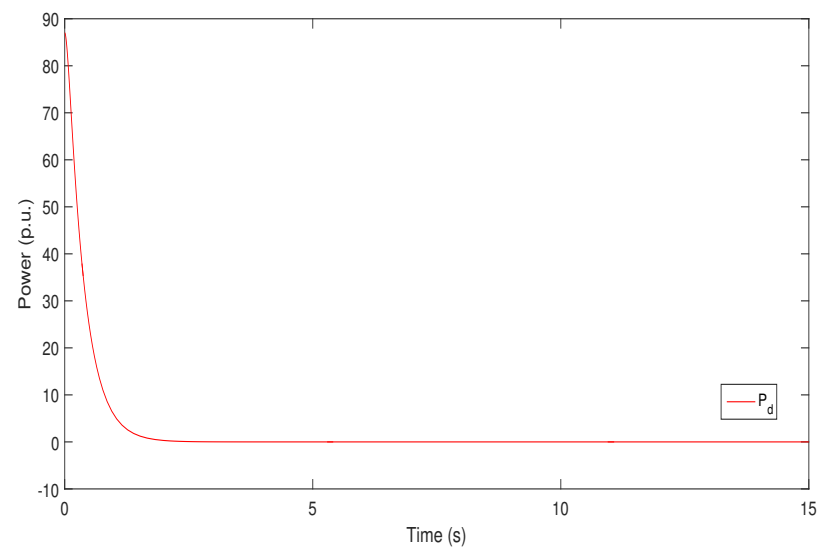

Fig. 5. The supply-demand mismatch update

\section{B. Case 5.2}

In this simulation study, the proposed algorithm is applied to a five-bus system in [39] by replacing the synchronous generator with a WT with a parallel-connected ESS in a directed communication network. The parameters of RGs and ESSs are selected according to [39]. The algorithm proposed in [39] is adopted in a network with bi-directional lines for agent's communication. As a result, the algorithm may lose its effectiveness if one of the bi-directional line breaks

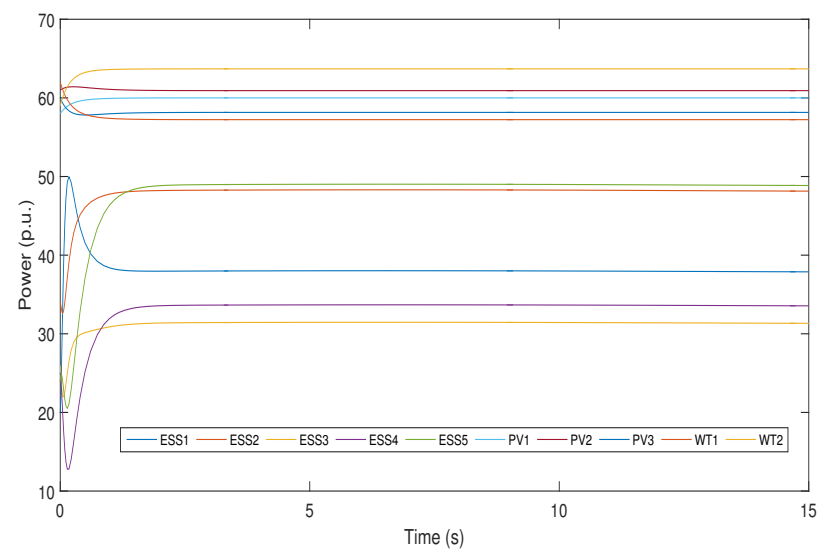

Fig. 6. The actual output power of RGs and ESSs

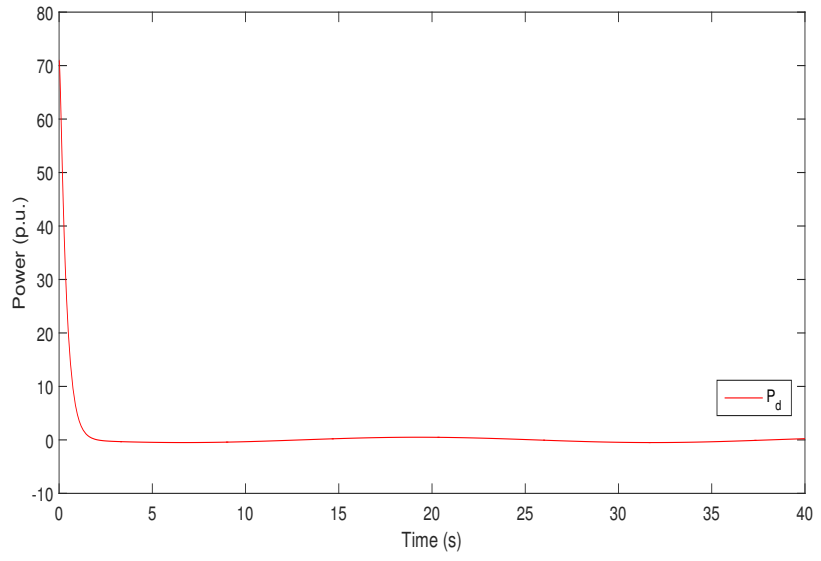

Fig. 7. The supply-demand mismatch update

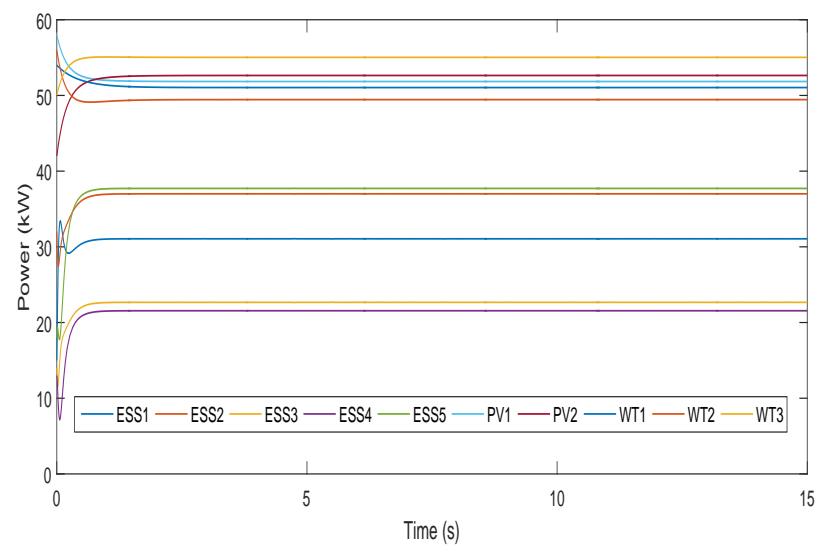

Fig. 8. The actual output power of RGs and ESSs

down. Instead of using bi-directional communication lines, our proposed algorithm can work under the directed graph that only needs directed communication lines, and the results are shown in Fig. 8 and Fig. 9. As the results shown, the power allocations of RGs and parallel-connected ESSs quickly converge to their optimal values, while the mismatch between supply and demand converges to zero, i.e., the optimization objective is achieved for this case.

\section{Case 5.3}

The robustness of single link/node failure is investigated in this case. In the first subcase, it is assumed that a breakdown of the transmission line for ESS3 at 10s, and it still can communicate with its neighbours. Because RGs are not used to tackle the demand mismatch, the rest of ESSs has to undertake the supply-demand balance in a fast response time. At 30s, the emergency line is employed, and hence ESS3 is plugged-in to support the load demand. The results are shown in Figs. 10 - 12. After ESS3 is pluged-out, the power allocations of all participants converge to the new optimal values. Further, the supply-demand mismatch can be handled by the rest of ESSs. When ESS3 is connected again, all the results converge to those of the previous ones.

Next, the second subcase considers when ESS3 fails to operate at $6 \mathrm{~s}$ and recovers at 40s. Upon failures, ESS3 loses 


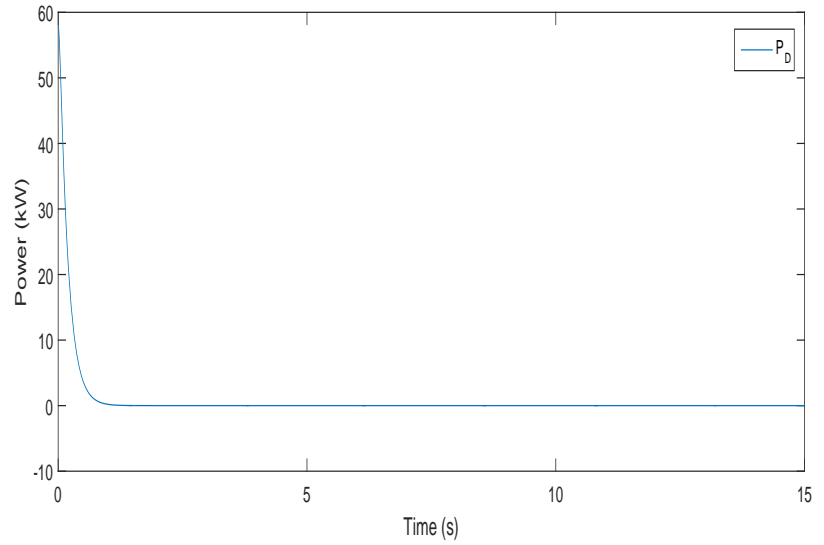

Fig. 9. The supply-demand mismatch update

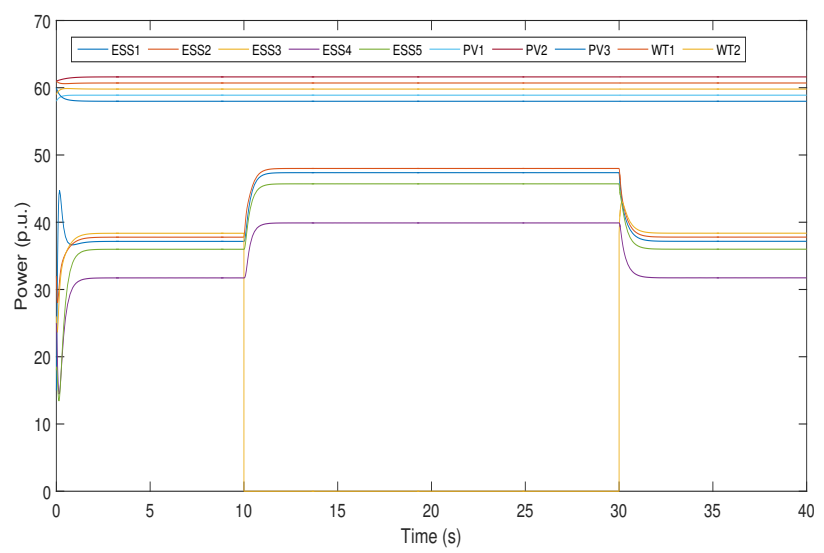

Fig. 10. The actual output power of RGs and ESSs during the single link failure

its communication with its neighbours, and the rest of ESSs is remain connected to support the load demand. The results in Fig. 13 and Fig. 14 show that the rest of the participants can converge to a new optimal operation condition, and the supplydemand mismatch is eliminated when ESS3 fails. Moreover, the results converge to those of the previous ones after ESS3 is repaired.

It should be noted that during the single link/node fail-

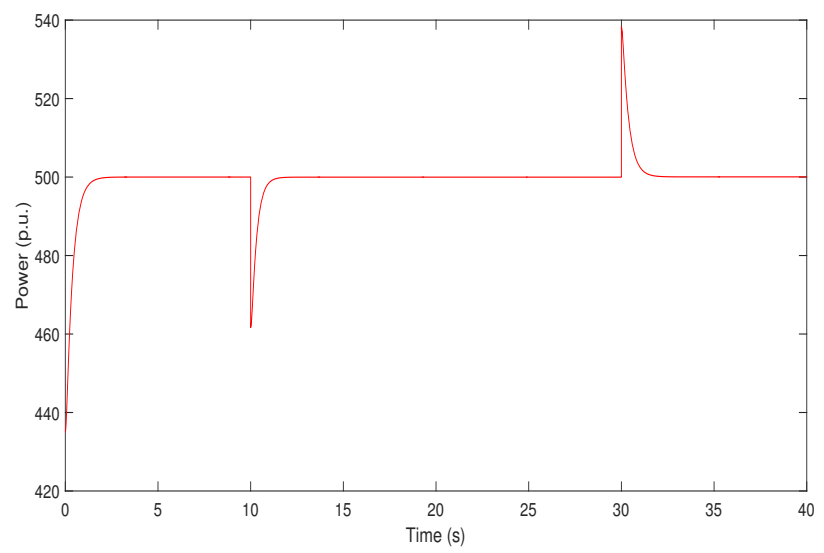

Fig. 11. The supply-demand mismatch update

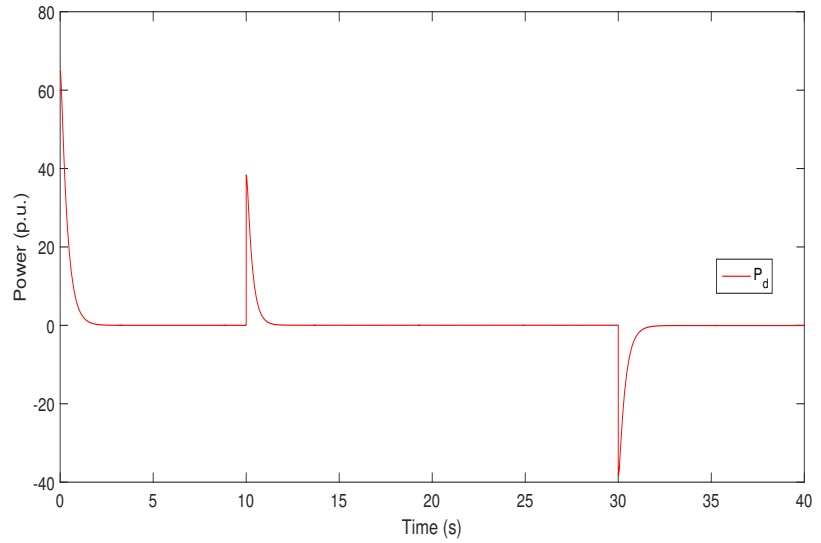

Fig. 12. Total allocated output power

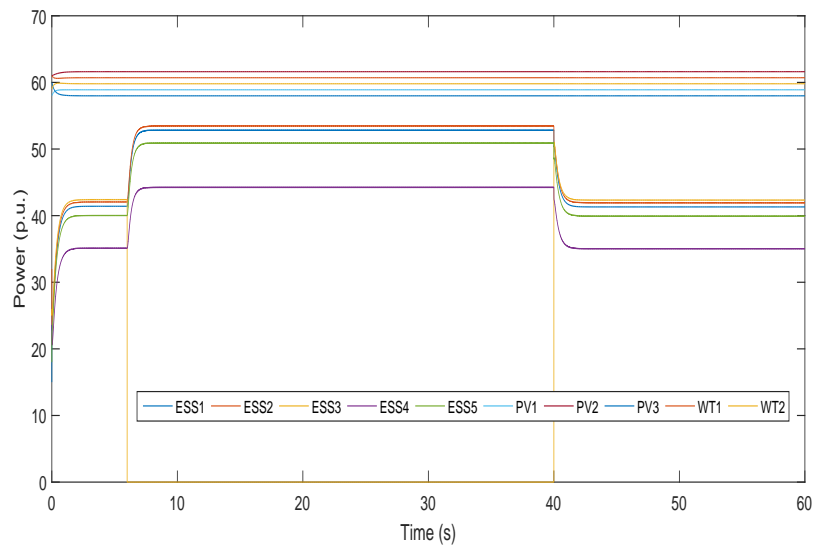

Fig. 13. The actual output power of RGs and ESSs during the single node failure

ure, the supply-demand deviations are small. Therefore, the proposed strategy will cause little effect on the frequency disturbance of the system, which may be applied to isolated systems such as an autonomous microgrid.

Remark 5.1: One of the necessary conditions for the convergence of the proposed algorithm is the connectivity of the communication network. Therefore, when the single-link/node failure occurred, the optimal solution is guaranteed if the communication network remains connected. Besides, the distributed information is used in the proposed algorithm instead of global information, which is robust to the link failures if the failing does not affect the connectivity of the communication network. Also, when one node fails to communicate with other neighbours, the rest group of nodes can continue their operations if the network is connected.

\section{Case 5.4}

The scalability of the proposed strategy is validated. To do so, we implement the optimal control strategy to the modified IEEE 162-bus system. There are three types of RGs in this system, i.e., eight PVs, eight WTs and one solar thermal, and two types of ESSs, i.e., battery storage system and fuel cells. The cost functions of fuel cell and solar thermal are based on the cost model in [44], [45], respectively. The communication network is weight-balanced and strongly connected. The initial 


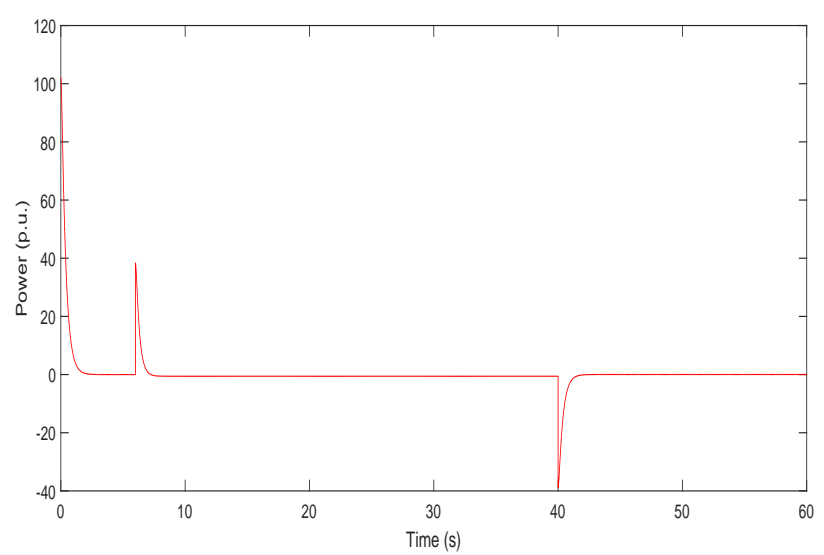

Fig. 14. The supply-demand mismatch update

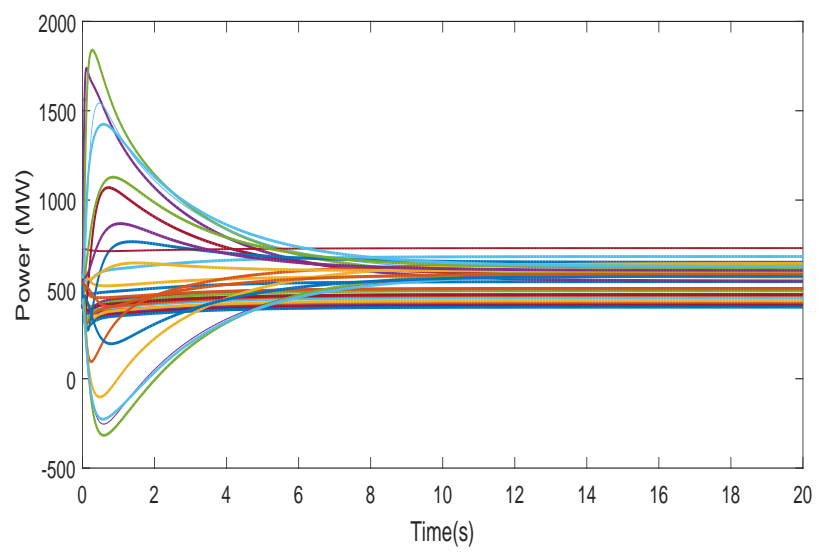

Fig. 15. The actual power output of RGs and ESSs

condition is given by [46] with a total load demand of 18422 MW.

As shown in Fig. 15 and Fig. 16, the proposed strategy can guarantee the allocated charging powers to converge to their optimal values within $12 \mathrm{~s}$ while the deviations of demand and supply power converge to zero, which demonstrates that the proposed algorithm is scalable.

Remark 5.2: It is noted that the proposed solution is compatible with diverse RGs and ESSs in the microgrid as

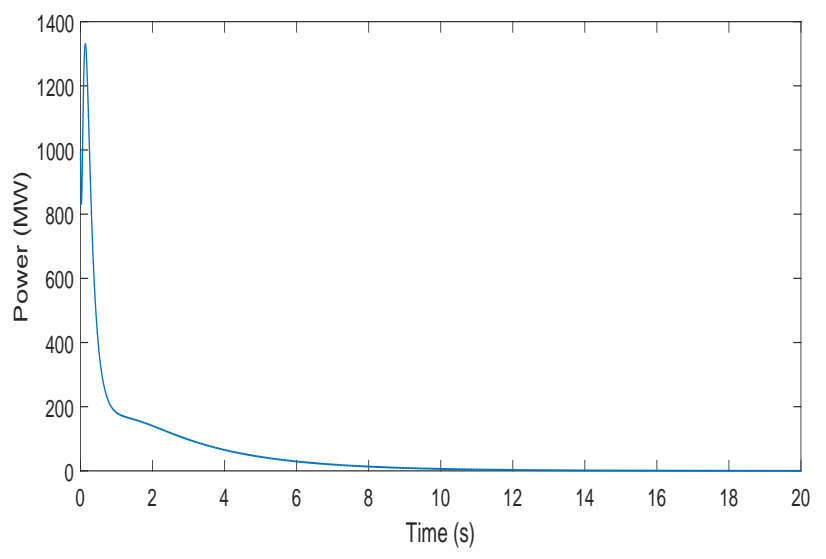

Fig. 16. The supply-demand mismatch update long as the cost function of RGs and ESSs can be formulated to satisfy the convex condition.

\section{CONCLUSION}

In this paper, a MAS-based distributed optimal strategy has been proposed to the optimal resource management in an islanded microgrid. The proposed strategy minimizes the generation cost of all participants, and meanwhile maintains the supply-demand equality condition within the microgrid system. The effectiveness of the proposed distributed strategy is demonstrated by the simulation using the IEEE test systems. Furthermore, the initializing procedures are no longer needed in the distributed solution design, and thus RGs and ESSs can start from any power allocation. The convergence of the proposed strategy only relies on a connected digraph, which demonstrates the robustness of single-link/node failures during the microgrid operation. In addition, the proposed distributed strategy can handle the time-varying supply-demand mismatch problem in isolated systems. To solve the resource management more realistic, practical constraints such as voltage limits, line capacities, network reconfiguration will be considered in our future work

\section{REFERENCES}

[1] F. Katiraei, R. Iravani, N. Hatziargyriou, and A. Dimeas, "Microgrids management," IEEE Power Energy Mag., vol. 6, no. 3, pp. 54-65, 2008.

[2] I. J. Balaguer, Q. Lei, S. Yang, U. Supatti, and F. Z. Peng, "Control for grid-connected and intentional islanding operations of distributed power generation," IEEE Trans. Ind. Electron., vol. 58, no. 1, pp. 147-157, 2011.

[3] Y. Zhang, N. Gatsis, and G. B. Giannakis, "Robust energy management for microgrids with high-penetration renewables," IEEE Trans. Sustain. Energy, vol. 4, no. 4, pp. 944-953, Oct 2013.

[4] M. Perrin, Y. Saint-Drenan, F. Mattera, and P. Malbranche, "Lead-acid batteries in stationary applications: competitors and new markets for large penetration of renewable energies," Journal of Power Sources, vol. 144, no. 2, pp. 402-410, 2005.

[5] X. Li, D. Hui, and X. Lai, "Battery energy storage station (BESS)-based smoothing control of photovoltaic (PV) and wind power generation fluctuations," IEEE Trans. Sustain. Energy, vol. 4, no. 2, pp. 464-473, April 2013.

[6] J. M. Eyer, J. J. Iannucci, and G. P. Corey, "Energy storage benefits and market analysis handbook, a study for the doe energy storage systems program," Sandia National Labratories, 2004.

[7] G. Bundestag, "Update of feed in tariff for renewable energies in Germany," Feed in international corporation, 2008.

[8] Y. Riffonneau, S. Bacha, F. Barruel, and S. Ploix, "Optimal power flow management for grid connected PV systems with batteries," IEEE Trans. Sustain. Energy, vol. 2, no. 3, pp. 309-320, 2011.

[9] F. Luo, K. Meng, Z. Y. Dong, Y. Zheng, Y. Chen, and K. P. Wong, "Coordinated operational planning for wind farm with battery energy storage system," IEEE Trans. Sustain. Energy, vol. 6, no. 1, pp. 253$262,2015$.

[10] K. Bradbury, L. Pratson, and D. Patiño-Echeverri, "Economic viability of energy storage systems based on price arbitrage potential in real-time US electricity markets," Applied Energy, vol. 114, pp. 512-519, 2014.

[11] A. J. Wood and B. F. Wollenberg, Power generation, operation, and control. John Wiley \& Sons, 2012.

[12] A. Chaouachi, R. M. Kamel, R. Andoulsi, and K. Nagasaka, "Multiobjective intelligent energy management for a microgrid," IEEE Trans. Ind. Electron., vol. 60, no. 4, pp. 1688-1699, 2013.

[13] B. Ramachandran, S. K. Srivastava, C. S. Edrington, and D. A. Cartes, "An intelligent auction scheme for smart grid market using a hybrid immune algorithm," IEEE Trans. Ind. Electron., vol. 58, no. 10, pp. 4603-4612, 2011

[14] N. Jain, S. Singh, and S. Srivastava, "A generalized approach for DG planning and viability analysis under market scenario," IEEE Trans. Ind. Electron., vol. 60, no. 11, pp. 5075-5085, 2013. 
[15] H. Xin, Z. Qu, J. Seuss, and A. Maknouninejad, "A self-organizing strategy for power flow control of photovoltaic generators in a distribution network," IEEE Trans. Power Syst., vol. 26, no. 3, pp. 1462-1473, 2011.

[16] M. G. Kallitsis, G. Michailidis, and M. Devetsikiotis, "Optimal power allocation under communication network externalities," IEEE Trans. Smart Grid, vol. 3, no. 1, pp. 162-173, 2012.

[17] Z. Wang, K. Yang, and X. Wang, "Privacy-preserving energy scheduling in microgrid systems," IEEE Transactions on Smart Grid, vol. 4, no. 4, pp. 1810-1820, 2013.

[18] A. De Bonis, J. P. Catalão, A. Mazza, G. Chicco, and F. Torelli, "A novel optimization algorithm solving network reconfiguration," in Power Systems Computation Conference (PSCC), 2014. IEEE, 2014, pp. 1-7.

[19] J.-H. Teng, S.-W. Luan, D.-J. Lee, and Y.-Q. Huang, "Optimal charging/discharging scheduling of battery storage systems for distribution systems interconnected with sizeable PV generation systems," IEEE Trans. Power Syst, vol. 28, no. 2, pp. 1425-1433, 2013.

[20] Z. Yang, R. Wu, J. Yang, K. Long, and P. You, "Economical operation of microgrid with various devices via distributed optimization," IEEE Trans. Smart Grid, vol. 7, no. 2, pp. 857-867, 2016.

[21] Y. Xu and Z. Li, "Distributed optimal resource management based on the consensus algorithm in a microgrid," IEEE Trans. Ind. Electron., vol. 62, no. 4, pp. 2584-2592, 2015.

[22] R. Olfati-Saber, J. A. Fax, and R. M. Murray, "Consensus and cooperation in networked multi-agent systems," Proceedings of the IEEE, vol. 95, no. 1, pp. 215-233, 2007.

[23] J. M. Solanki, S. Khushalani, and N. N. Schulz, "A multi-agent solution to distribution systems restoration," IEEE Trans. Power syst, vol. 22, no. 3, pp. 1026-1034, 2007.

[24] W. Liu, W. Gu, W. Sheng, X. Meng, Z. Wu, and W. Chen, "Decentralized multi-agent system-based cooperative frequency control for autonomous microgrids with communication constraints," IEEE Trans. Sustain. Energy, vol. 5, no. 2, pp. 446-456, April 2014.

[25] B. Zhao, C. Guo, and Y. Cao, "A multiagent-based particle swarm optimization approach for optimal reactive power dispatch," IEEE Trans. Power Syst, vol. 20, no. 2, pp. 1070-1078, 2005.

[26] W. Ren and R. W. Beard, "Consensus seeking in multiagent systems under dynamically changing interaction topologies," IEEE Transactions on automatic control, vol. 50, no. 5, pp. 655-661, 2005.

[27] C. Zhao, J. He, P. Cheng, and J. Chen, "Consensus-based energy management in smart grid with transmission losses and directed communication," IEEE Transactions on Smart Grid, 2016.

[28] Z. Ding and Z. Li, "Distributed adaptive consensus control of nonlinear output-feedback systems on directed graphs," Automatica, vol. 72, pp. 46-52, 2016.

[29] F. Bullo, J. Cortés, and S. Martinez, Distributed control of robotic networks: a mathematical approach to motion coordination algorithms. Princeton University Press, 2009.

[30] W. D. Kellogg, M. H. Nehrir, G. Venkataramanan, and V. Gerez, "Generation unit sizing and cost analysis for stand-alone wind, photovoltaic, and hybrid wind/PV systems," IEEE Trans. Energy Convers., vol. 13, no. 1, pp. 70-75, Mar 1998.

[31] A. D. Dominguez-Garcia, S. T. Cady, and C. N. Hadjicostis, "Decentralized optimal dispatch of distributed energy resources," in 2012 IEEE 51st IEEE Conference on Decision and Control (CDC). IEEE, 2012, pp. 3688-3693.

[32] R. Deng, Z. Yang, J. Chen, and M.-Y. Chow, "Load scheduling with price uncertainty and temporally-coupled constraints in smart grids," IEEE Trans. Power Syst., vol. 29, no. 6, pp. 2823-2834, 2014.

[33] Z. Wang, W. Wu, and B. Zhang, "A fully distributed power dispatch method for fast frequency recovery and minimal generation cost in autonomous microgrids," IEEE Trans. Smart Grid, vol. 7, no. 1, pp. 19-31, Jan 2016.

[34] T. Zhao, Z. Zuo, and Z. Ding, "Cooperative control of distributed battery energy storage systems in microgrids," in 2016 35th Chinese Control Conference (CCC), July 2016, pp. 10019-10024.

[35] F. A. Amoroso and G. Cappuccino, "Advantages of efficiency-aware smart charging strategies for pevs," Energy Conversion and Management, vol. 54, no. 1, pp. 1-6, 2012.

[36] D. P. Bertsekas, "Necessary and sufficient conditions for a penalty method to be exact," Math. Programming, vol. 9, no. 1, pp. 87-99, 1975.

[37] S. Li and Y. Guo, "Dynamic consensus estimation of weighted average on directed graphs," International Journal of Systems Science, vol. 46 , no. 10, pp. 1839-1853, 2015.

[38] Z. Ding, Nonlinear and Adaptive Control Systems. London, U.K.: Inst. Eng. Technol. (IET), 2013.
[39] Y. Xu, W. Zhang, G. Hug, S. Kar, and Z. Li, "Cooperative control of distributed energy storage systems in a microgrid," IEEE Trans. Smart Grid, vol. 6, no. 1, pp. 238-248, 2015.

[40] F. Chen, M. Chen, Q. Li, K. Meng, Y. Zheng, J. Guerrero, and D. Abbott, "Cost based droop schemes for economic dispatch in islanded microgrids," 2016.

[41] G. Binetti, A. Davoudi,F. L. Lewis, D. Naso and B. Turchiano “ Distributed Consensus-Based Economic Dispatch With Transmission Losses," IEEE Trans. Power Syst, vol. 29, no. 4, pp. 1711-1720, 2014.

[42] Y. Xu, W. Zhang, W. Liu, X. Wang, F. Ferrese, C. Zang, and H. Yu, "Distributed subgradient-based coordination of multiple renewable generators in a microgrid," IEEE Trans. Power Syst, vol. 29, no. 1, pp 23-33, 2014.

[43] N. Rahbari-Asr and M.-Y. Chow, "Cooperative distributed demand management for community charging of PHEV/PEVs based on KKT conditions and consensus networks," IEEE Trans. Ind. Informat., vol. 10 , no. 3, pp. 1907-1916, 2014.

[44] X. Hu,, N. Murgovski, L. M. Johannesson, and B.Egardt, "Optimal dimensioning and power management of a fuel cell/battery hybrid bus via convex programming," IEEE/ASME transactions on mechatronics, vol. 20, no. 1, pp. 457-468, 2015.

[45] M.T. Ameli, S. Moslehpour, and M. Shamlo, "Economical load distribution in power networks that include hybrid solar power plants, " Electric Power Systems Research, vol. 78, no. 7, pp. 1147-1152, 2008.

[46] P. S. T. C. Archive:, "162-bus, 17 generator dynamic test case," http: //www.ee.washington.edu/research/pstca/dyn17/pg_tcadd17.htm.

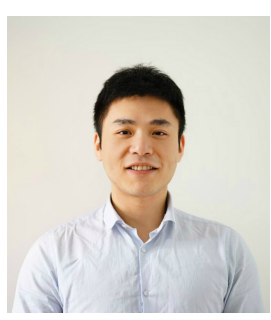

Tianqiao Zhao received his B.Eng. degree in automatic control from North China Electric Power University, Hebei, China, in 2013, and his M.Sc degree in electrical and electronic engineering from the University of Manchester, U.K., in 2014. He is now a Ph.D. candidate in control engineering with the School of Electrical and Electronic Engineering at the University of Manchester, U.K. His research interests include distributed optimization of microgrids, distributed control and energy storage systems.

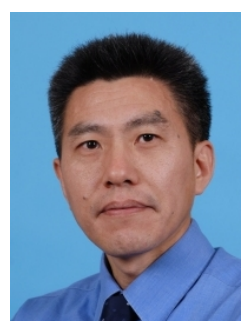

Zhengtao Ding received the B.Eng. degree from Tsinghua University, Beijing, China, and the M.Sc. degree in systems and control and the Ph.D. degree in control systems from the University of Manchester Institute of Science and Technology, Manchester, U.K. After working as a Lecturer with Ngee Ann Polytechnic, Singapore, for ten years, in 2003, he joined The University of Manchester, Manchester, U.K., where he is currently a Senior Lecturer of control engineering with the School of Electrical and Electronic Engineering. He is the author of the book Nonlinear and Adaptive Control Systems (IET, 2013) and a number of journal papers. His research interests include nonlinear and adaptive control theory and their applications. Dr. Ding serves as an Associate Editor for the IEEE Transactions on AUtOMATIC CONTROL, Transactions of the Institute of Measurement and Control, Control Theory and Technology, Mathematical Problems in Engineering, Unmanned Systems, and the International Journal of Automation and Computing. 